\title{
Development of a Compact Wide-Slot Antenna for Early Stage Breast Cancer Detection Featuring Circular Array Full-View Geometry
}

\author{
S. S. Tiang, M. S. Hathal, N. S. Nik Anwar, M. F. Ain, and M. Z. Abdullah \\ School of Electrical and Electronic Engineering, Universiti Sains Malaysia, Engineering Campus, 14300 Penang, Malaysia \\ Correspondence should be addressed to M. Z. Abdullah; mza@usm.my
}

Received 16 September 2013; Revised 14 May 2014; Accepted 19 May 2014; Published 10 June 2014

Academic Editor: Atsushi Mase

Copyright (c) 2014 S. S. Tiang et al. This is an open access article distributed under the Creative Commons Attribution License, which permits unrestricted use, distribution, and reproduction in any medium, provided the original work is properly cited.

A novel planar type antenna printed on a high permittivity Rogers' substrate is proposed for early stage microwave breast cancer detection. The design is based on a p-shaped wide-slot structure with $50 \Omega$ microstrip feeding circuit to eliminate losses of transmission. The design parameters are optimized resulting in a good reflection coefficient at $-10 \mathrm{~dB}$ from 4.5 to $10.9 \mathrm{GHz}$. Imaging result using inhomogeneous breast phantom indicates that the proposed antenna is capable of detecting a $5 \mathrm{~mm}$ size cancerous tumor embedded inside the fibroglandular region with dielectric contrast between the target and the surrounding materials ranging from $1.7: 1$ to $3.6: 1$.

\section{Introduction}

Since the Federal Communication Commission (FCC) has allocated the frequency band $3.1-10.6 \mathrm{GHz}$ for commercial application [1], the ultra-wideband (UWB) systems have been widely used worldwide particularly for imaging systems. UWB signal is a short pulse with subnanosecond order, which is located in the spectrum below the acceptable noise level. It is also widely used for applications involving short-range very high-speed data transfer like the broadband access to the internet, covert communication, localization and asset tracking, and so forth. UWB pulses are generally shorter than the target dimension, making it an attractive tool for biomedical applications like nondestructive visualization of human organs. Another characteristic of UWB is its ability to resolve an object with dimension exceeding Rayleigh's limit, leading to accuracy approaching the submillimeter resolution in fatty dielectrics [2]. UWB also exhibits strong multipath, temporal, and space resolving capability, making it an ideal candidate for localization and detection of cancerous tumor at the early stage of the disease.

Since UWB transmits narrow short pulses instead of continuous wave, therefore, a reasonably wideband antenna is needed in order to capture the important information of the scatterers in the time domain. Conventional UWB antennas have been introduced for this application such as dipole [3], resistively loaded bowtie [4], and Vee dipoles [5]. However, resistively loaded antennas have inherent drawbacks in terms of size, bandwidth, and pulse distortion. One major limitation of the conventional designs was their narrow bandwidth. This limitation was successfully overcome with the progress of UWB antenna research pursued by many groups worldwide. A modified pyramidal horn operating from 1 to $11 \mathrm{GHz}$ has been investigated at the University of Wisconsin-Madison [6]. In spite of a good impedance matching and fidelity, its efficiency is low due to resistive loading. Another UWB antenna belonging to antipodal type for the breast cancer imaging was studied by a research group at the University of Queensland [7]. This tapered slot antenna has an impedance bandwidth of $2.75-11 \mathrm{GHz}$ with an average size of $50 \mathrm{~mm} \times$ $50 \mathrm{~mm}$. Although this design reasonably exhibits broadband characteristic, the antenna requires larger geometry and is difficult to build. Therefore, this type of antenna is not well-suited for array applications such as the tomographic measurement. A stacked-patch antenna with a microstrip line fed through a slot in the ground plane was also studied at the University of Bristol [8]. This design has a $-10 \mathrm{~dB}$ impedance bandwidth from 4 to $9.5 \mathrm{GHz}$ with a small mismatch in the 


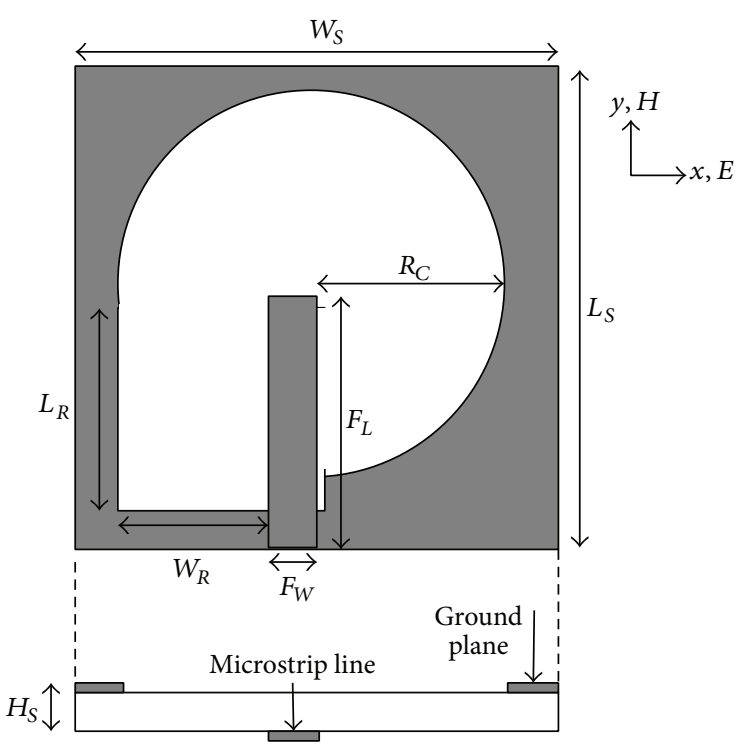

(a)

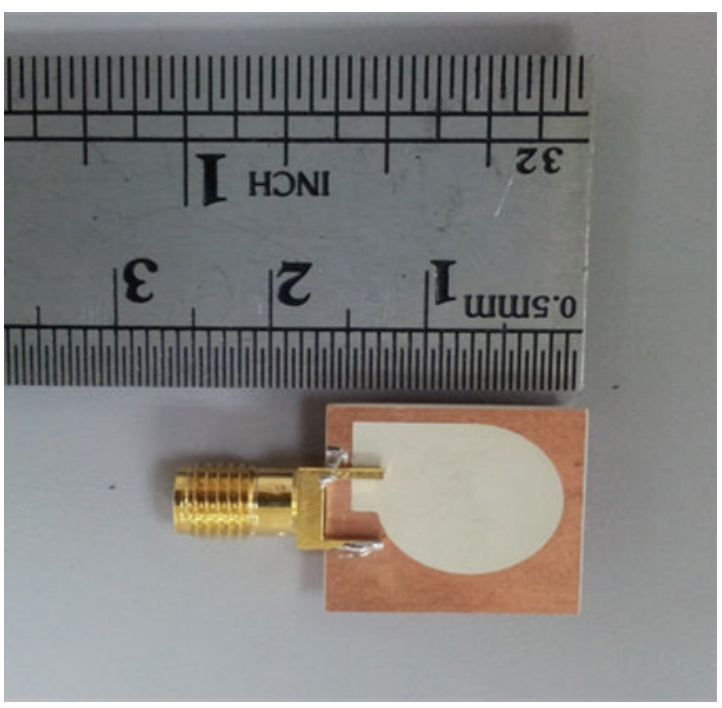

(b)

Figure 1: The proposed p-shaped wide-slot antenna. (a) The schematic and (b) the actual designs.

mid of the spectrum. Although the largest patch of this design is only $6.5 \mathrm{~mm} \times 9 \mathrm{~mm}$, the whole antenna structure is still bulky due to feeding line and ground plane. In summary, all the existing designs are a result of trade-off between size and bandwidth. Some of the advantages of the previously reported UWB structures are stronger radiations, higher gain, and good directivity over most of its intended frequency. The main drawback of the existing configurations is the element size which is relatively large. Hence, the design of a compact UWB antenna with reasonably large bandwidth continues to attract biomedical engineers and scientists dealing with applications involving human organs, particularly the breast cancer detection.

For breast imaging, a planar-type design is preferred as the antenna can be placed directly on the target. In addition to wideband characteristic, this antenna should also preferably be small in size, lightweight, and compact. One new emerging design which fulfills these requirements is the microstrip printed slot antennas. Due to its favorable impedance characteristics, this type of antenna, particularly the printed wide-slot antenna, has been designed and evaluated in the laboratory for medical imaging. Slot antennas provide large magnetic fields which are less prone to near-field coupling with nearby objects [9]. Recently, different printed wideslot antennas fed by a microstrip line or coplanar waveguide have been proposed $[10,11]$. A fork-fed wide-slot antenna intended for UWB antenna array application has recently been demonstrated [12]. By using different feeding techniques or employing different slot shapes, different types of slot antennas have been designed to achieve ultra-wideband performance [13-15]. The shape of the wide-slot can be square, rectangular, triangular, circular, elliptical, and so forth. Meanwhile the typical feeding structures can be shaped as cross, fork, triangle, square, arc, and so forth. Wideslot antenna with an open-ended feed structure, covering the frequency range from 3.1 to $10.6 \mathrm{GHz}$, has also been reported [16]. The bandwidth of the wide-slot antenna is dependent on the geometrical parameters of the slot and the feeding structure. Though promising, however, most of the existing wide-slot designs suffer several drawbacks due to their limited impedance bandwidth and bulky configuration. In addition, variation of the electrical length of antennas with frequency causes significant distortion in the radiated waveforms. In addressing some of these shortcomings, a pshaped wide-slot antenna with simple tuning stub has been designed and fabricated in our laboratory. The $16 \mathrm{~mm} \times$ $16 \mathrm{~mm}$ size antenna, whose dimension was optimized to cover the frequency range from 4.5 to $10.9 \mathrm{GHz}$, was designed and fabricated using Rogers' type printed circuit board. Important characteristics like the antenna reflection coefficient, transmission coefficient, and fidelity are validated experimentally. For the purpose of imaging the design was used together with the improved version of the beamforming image reconstruction algorithm referred herein as the enhanced delay and sum (EDAS) [17]. The sensors and instrumentation were tested using chemically derived heterogeneous breast phantom, simulating cancerous and normal tissues in an average female adult at the early stage of the disease.

\section{UWB Circular Antenna Array and Measurement Setup}

2.1. Antenna Design. The geometry and configuration of the proposed antenna are shown in Figure 1. The antenna consists of a p-shaped slot radiator etched out of the ground plane on one side of a dielectric Rogers' substrate RT6010, with relative permittivity 10.2 and a $50 \Omega$ feeding line, forming a microstrip line tuning stub along the $y$-axis. The slot radiator in the 


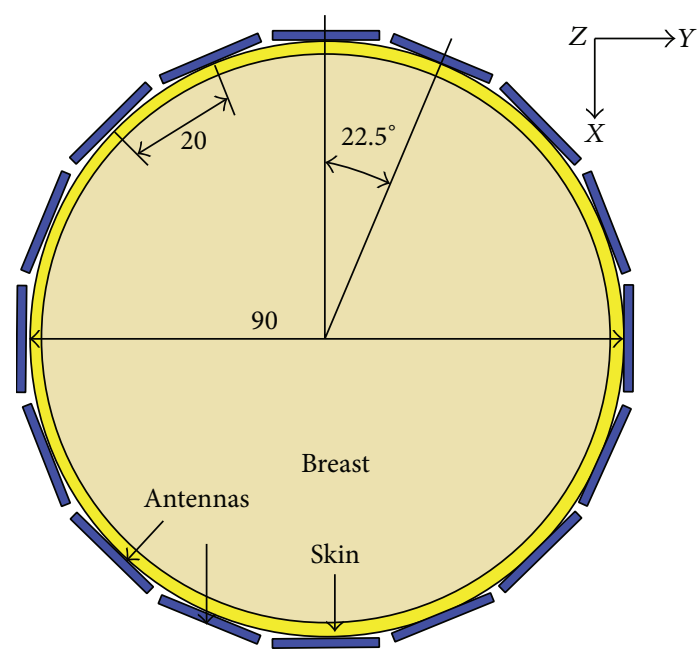

FIGURE 2: Circular antenna array for full view geometry.

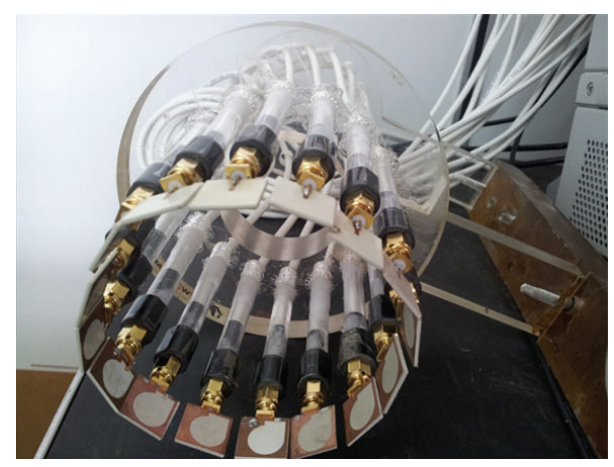

FIGURE 3: Actual design of the antenna holder.

ground plane has strong coupling to the feeding structure. The microstrip line having a width $\left(F_{W}\right)$ of $1.2 \mathrm{~mm}$ and length $\left(F_{L}\right)$ of $7.5 \mathrm{~mm}$ is connected to the coaxial cable through an SMA connector. The radiating structure occupies an area of $16 \mathrm{~mm} \times 16 \mathrm{~mm}$ with a height $\left(H_{S}\right)$ of $1.27 \mathrm{~mm}$. The design includes a primary circular slot of radius $6 \mathrm{~mm}$ and a secondary rectangular slot of dimension $L_{R} \times W_{R}$. Compared with the existing designs, the proposed structure has a simple design due to less number of parameters. The detailed dimensions of the proposed antenna are summarized in Table 1. The electromagnetic solver, CST Microwave Studio, is used to numerically investigate and optimize the design. Parametrically, it will be shown in this paper that the proposed antenna achieves a good impedance matching and a flat magnitude of transmission coefficient over the operating frequency range.

2.2. Circular Array. In this paper, we present a radarbased breast cancer detection system. It uses a circular antenna array and a breast phantom model with dielectric properties similar to real breast tissues. Our experimental system was built in such way that it can be used directly with real breast cancer patients in future work. The array is formed by 16 proposed antennas, placed equidistantly

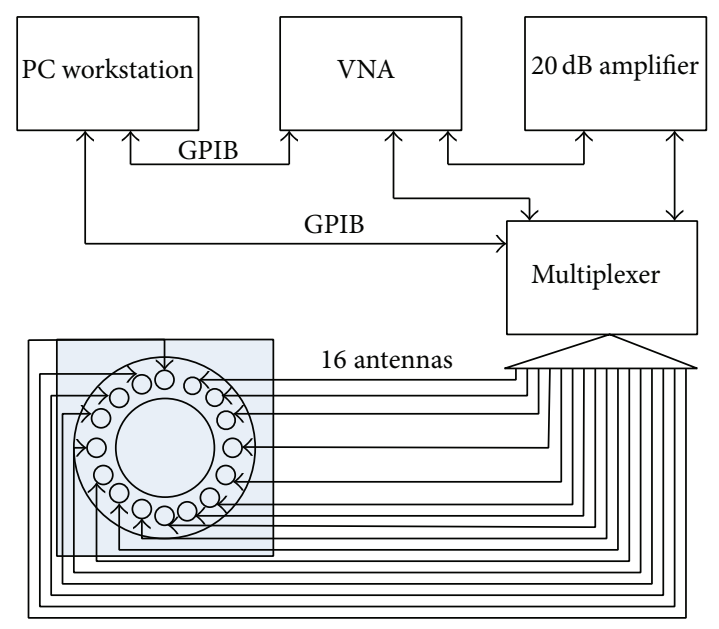

FIGURE 4: Experiment setup of the imaging system.

TABLE 1: Optimized design parameters.

\begin{tabular}{lcccccccc}
\hline Parameters & $R_{C}$ & $L_{R}$ & $W_{R}$ & $F_{L}$ & $F_{W}$ & $L_{S}$ & $W_{S}$ & $H_{S}$ \\
Unit $(\mathrm{mm})$ & 6 & 6.5 & 7.2 & 7.5 & 1.2 & 16 & 16 & 1.27 \\
\hline
\end{tabular}

around the breast phantom, making an angle of $22.5^{\circ}$ from the origin. The breast phantom consists of a $2 \mathrm{~mm}$ thick layer of skin and $88 \mathrm{~mm}$ diameter of the breast. In this geometry the longest and shortest sensing distances correspond to two opposite and adjacent antennas, respectively. The distance between any two opposing antennas is $90 \mathrm{~mm}$ whereas the distance of any two adjacent antennas is approximately $20 \mathrm{~mm}$. Figure 2 shows the circular antenna array and the configuration of breast phantom in full-view geometry.

The data acquisition system used is based on the 16curved circular array, surrounding the target. All the 16 antennas are driven via a $50 \Omega$ coaxial feeder, incorporating in-line ferrite toroid to ensure balanced antenna feeding, and through which the RG 54 coaxial cable is threaded. Figure 3 shows the actual design of the antenna holder and the cabling system, connecting each antenna to a multiplexer. Figure 4 shows the experimental setup. The data acquisition system consists of a Cytec CXM 32 multiplexer that automatically switches transmitting and receiving lines. The multiplexer is connected to an Agilent E5071C vector network analyzer (VNA) providing the measurements in time domain. Also, a wide band $20 \mathrm{~dB}$ amplifier is used to improve the received signal power.

2.3. Breast Phantom Construction. To experiment the methods for breast cancer detection, the heterogeneous glandular model was constructed using chemical materials which are formulated based on the relative permittivity and conductivity of realistic human tissues over the frequency of interest given in Table 2 [18]. In the fabrication the chemical compositions are varied so that the electrical characteristics (permittivity, conductivity, and dispersion) of resulting mixtures 
TABLE 2: Relative permittivity and conductivity of breast tissue at frequency range from $200 \mathrm{MHz}$ to $5 \mathrm{GHz}$ [18].

\begin{tabular}{lcc}
\hline Tissue & Relative permittivity & Conductivity \\
\hline Fat & 15 & 0.15 \\
Skin & 38 & 1.9 \\
Glandular & 32 & 1.5 \\
Tumour & 54 & 2.5 \\
\hline
\end{tabular}

closely matched with the breast tissues at the required signal bandwidth. In this paper, an oil-gelatin mixture is used as a phantom material which is based on heterogeneous dielectric permittivity of breast tissues available from the literature [19]. Figure 5 shows the chemical-based heterogeneous phantom that consists of a $2 \mathrm{~mm}$ thick skin layer, $88 \mathrm{~mm}$ diameter fatty tissue, and two $20 \mathrm{~mm}$ radius circular shaped glandular structures, with relative permittivity of 38,15 , and 32 , respectively. A $5 \mathrm{~mm}$ target with relative permittivity of 54 is inserted in one of these glandular structures, simulating the cancerous tumor at the very early stage of development. Data acquired are processed using EDAS, from which an image depicting the energy distribution inside the medium is reconstructed in 2 dimensions.

\section{Image Reconstruction Algorithm}

UWB radar imaging aims to reconstruct the presence and location of the target arising from the dielectric contrast between healthy and malignant tissues. The qualitative image is displayed as a variance of energy intensity. Figure 6 shows the sensing geometry of 16 transceivers locations. For UWB radar sensing, a transmitting antenna $(T \mathrm{x})$ sends a very short pulse that penetrates into the region under test, and it will be scattered by any target that exhibits variation contrast in dielectric properties, whereas the receiving antennas $(\mathrm{Rx})$ record these scattered signals from which the target nonhomogeneities are reconstructed. A total of $M(N-1)$ independent measurements are captured from a radar system which comprises $M$ transmitters and $N$ receivers.

The image reconstruction algorithm investigated in this application is similar to delay and sum (DAS) algorithm which is among the earliest qualitative radar techniques developed for breast cancer detection [20]. Herein the resulting algorithm is referred to as the enhanced delay and sum (EDAS). The aim of EDAS is primarily to create a pure response signal of the tumor from measured data. Principally the received information includes backscattered signals from malignant breast tissues and clutter (late arrivals) in addition to the backscattered from skin-breast interface and the input signal (early arrivals). Signal processing is used to remove the early arrivals, which dominate the backscattered signals, and filtering them will lead to improving the signal-to-noise ratio of the tumor response. An averaging artifact removal procedure is used to remove the skin artifacts. These artifacts are removed using a calibration process that avoids the use of a breast phantom or a priori information. In so doing, a reference signal is firstly generated by averaging all recorded signals as follows:

$$
k(t)=\frac{1}{M N} \sum_{m=1}^{M} \sum_{n=1}^{N} x_{m, n}(t),
$$

where $x_{m, n}(t)$ is the recorded backscattered signal from $m$ th transmitter and $n$th receiver. Then the reference signal is subtracted from each of the backscattered signals, resulting in the calibrated signals, $x_{m n}^{\prime}(t)$ which contains only the tumor response and clutters. Mathematically

$$
x_{m, n}^{\prime}(t)=k(t)-x_{m, n}(t) .
$$

The time delay for all recorded signals is then calculated for all transmitter and receiver locations and for each focal point, $r$, as follows:

$$
\tau_{m, n}(r)=\frac{\left\|r-r_{m}\right\|+\left\|r-r_{n}\right\|}{v \Delta t},
$$

where the speed of propagation is given by

$$
v=\frac{c}{\sqrt{\varepsilon_{r}}}
$$

and $\left\|r_{m}-r\right\|$ and $\left\|r_{n}-r\right\|$ are the distances between the focal point $r$ and $m$ th transmitter and $n$th receiver, $\Delta t$ is the sampling time, $c$ is the velocity of light in vacuum, and $\varepsilon_{r}$ is the relative permittivity of the medium. Each calibrated signal in (2) is time shifted with an appropriate delay time calculated in (3).

Following time alignment, a focusing-quality procedure utilizing the coherence factor (CF) is implemented. Traditionally, the CF is used for anomaly correction and side lobe suppression in ultrasound imaging applications [21]. This procedure is based on utilization of the coherence quality weighting of the backscattered signals at specific focal points inside the domain. The same method can be applied for UWB imaging, which could significantly enhance the image as results from ultrasonic experiments suggest [22]. In this case, the coherence factor is defined as the ratio between the energy of the coherent sum and the total incoherent energy. Using (2) and (3), the coherence factor $\mathrm{CF}(r)$ is calculated as follows:

$$
\begin{aligned}
\mathrm{CF}(r) & =\frac{\text { coherent energy }}{\text { total incoherent energy }} \\
& =\frac{\left|\sum_{m=1}^{M} \sum_{n=1}^{N} x_{m, n}^{\prime}\left(t-\tau_{m, n}(r)\right)\right|^{2}}{\sum_{m=1}^{M} \sum_{n=1}^{N}\left|x_{m, n}^{\prime}\left(t-\tau_{m, n}(r)\right)\right|^{2}}
\end{aligned}
$$

The focusing quality $\mathrm{FQ}_{m, n}(r)$ is then obtained by multiplying the coherence factor with the calibrated signal after time-shifting. Mathematically

$$
\mathrm{FQ}_{m, n}(r)=\mathrm{CF}(r) x_{m, n}^{\prime}\left(t-\tau_{m, n}(r)\right)
$$

The high-coherence signals (high coherence factor) represent the main lobe part, which creates an image with good 


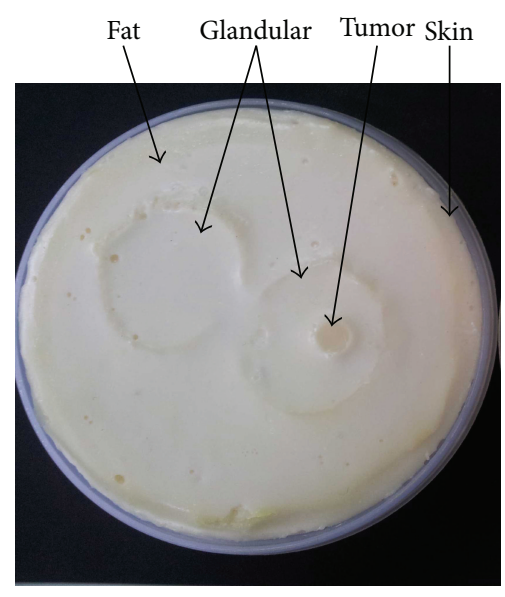

(a)

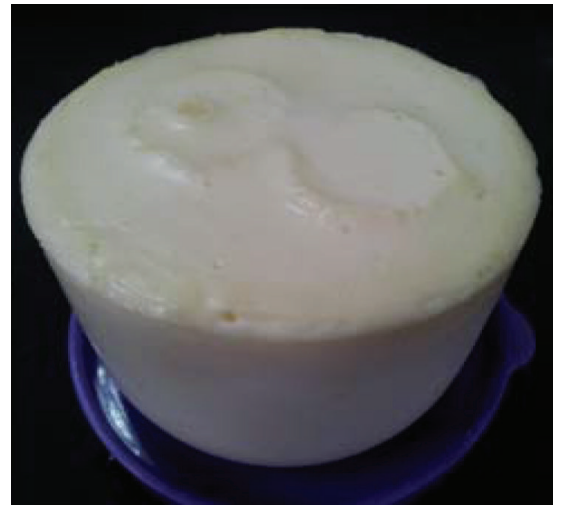

(b)

Figure 5: The realistic chemical-based heterogeneous breast phantom. (a) Top view. (b) Side view.

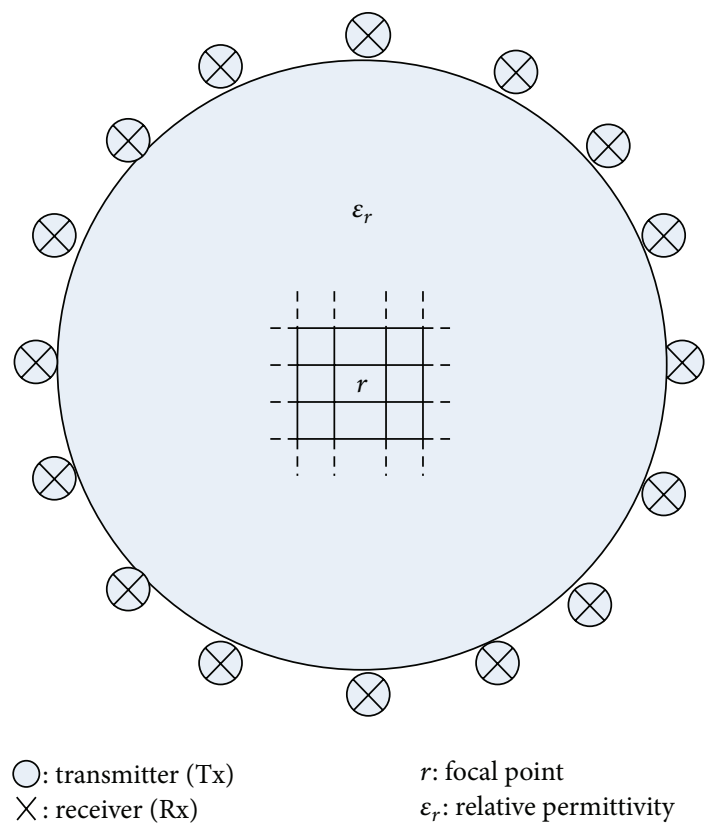

FIGURE 6: Sensing geometry of multistatic aperture radar with 16 antennas.

focusing quality, and their amplitude should be maintained. On the other hand, the incoherent signals (low coherence factor) represent the side lobe part, which creates an image with poor focusing quality, and their amplitude should be suppressed. Consequently, the amplitude of each image pixel is adaptively weighted by the corresponding CF such that the unwanted side lobes are effectively reduced.

The enhancement in tumor detection capability of EDAS algorithm is based on the supplementary weighting process involving pairing multiplication of the backscattered signals. In this case, each signal pair measured from the same transmitter is multiplied with one another and their products are summed. The results from these procedures are squared and then integrated over a window length, $w$, producing the energy at each focal point. Mathematically

$$
E(r)=\int_{0}^{w}\left[\sum_{m=1}^{M} \sum_{n=1}^{N-1} \mathrm{FQ}_{m, n}(r) \mathrm{FQ}_{m, n+1}(r)\right]^{2} d t .
$$

The assembly of $E(r)$ at each focal point produces the desired image, depicting the distributions of energy reflected by the scatterer.

\section{Results and Discussion}

In order to investigate the performance and establish some salient characteristics of the proposed design, series of simulated experiments were firstly performed and, secondly, followed by laboratory experiments. Image reconstruction experiment was also performed in order to assess the feasibility of applying the overall system for breast cancer detection.

4.1. Antenna Simulation and Measurement. A few sensitive design parameters were investigated numerically in order to study antenna characteristics and behavioral performance across the entire band. Wide-slot antenna is well known for its wide impedance bandwidth; however, it is restricted by the degradation of the radiation patterns at higher frequencies. In this particular design the bandwidth of the antenna is determined by the shapes and sizes of the slot.

Figure 7 shows the reflection coefficient, $S_{11}$, for different shapes of slot. Clearly, the circular-shaped slot and the elliptical-shaped slot provide limited narrow bandwidth, averaging at $51 \%$ and $62 \%$ in the frequency range from 6.4 to $10.8 \mathrm{GHz}$ and from 5.3 to $10.1 \mathrm{GHz}$, respectively. Meanwhile, the rectangular-shaped slot has an impedance bandwidth with large discrepancies where $S_{11}$ rises above $-10 \mathrm{~dB}$ from 6 to $8.5 \mathrm{GHz}$. Compared to these three designs, noticeably, the p-shaped slot provides much wider bandwidth, averaging at $83 \%$ in the frequency range $4.5-10.9 \mathrm{GHz}$. The increase in bandwidth is partly due to changes in the electric field distribution due to the presence of $\mathrm{p}$-shaped slot which reduces the 


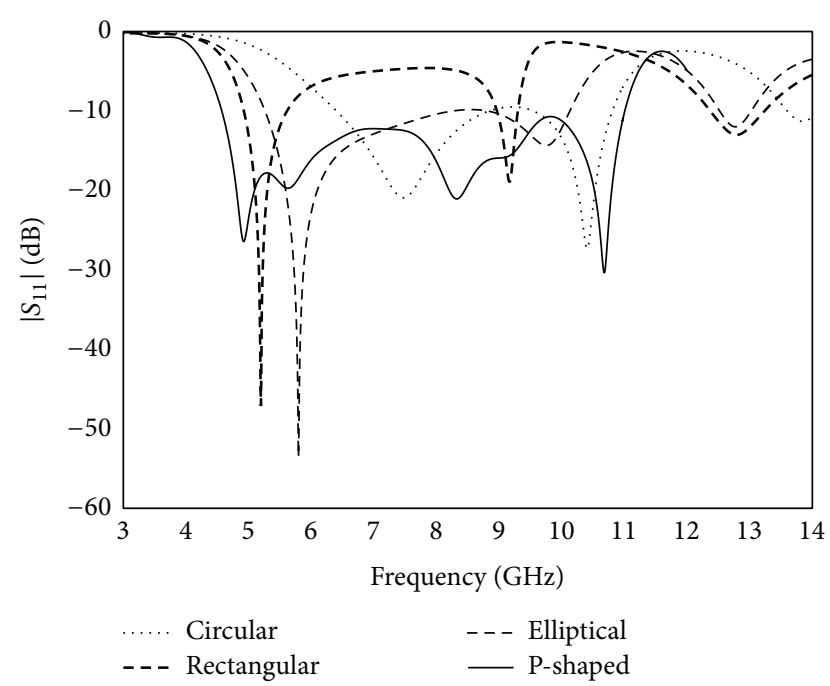

Figure 7: Simulated reflection coefficient for different slot shapes.

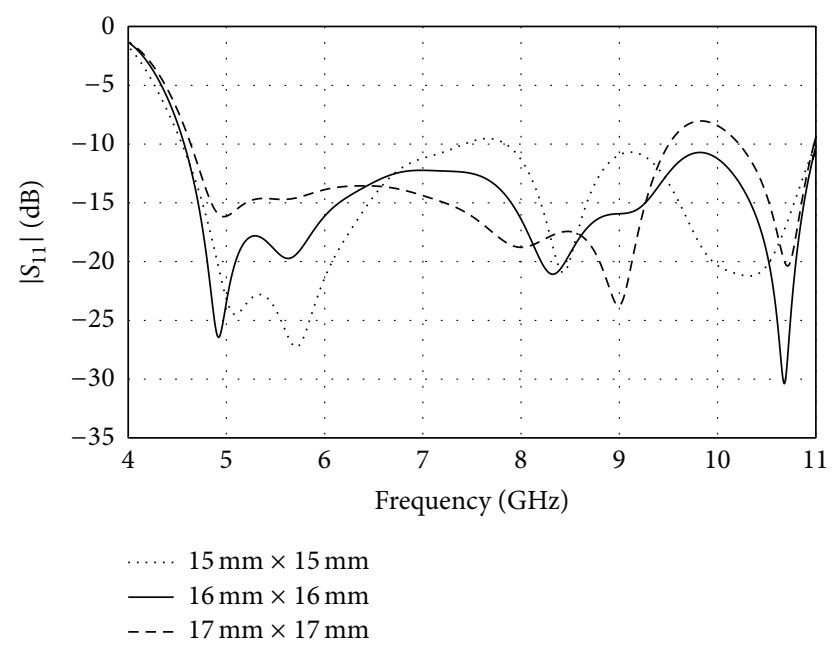

FIGURE 8: Simulated reflection coefficient for various substrate sizes.

longest current path. Resultantly, the impedance matching is significantly improved, especially at lower frequencies as evident in Figure 7. Hence, a microstrip line fed with a pshaped slot can provide wider bandwidth compared to other designs.

Another factor which has increased the impedance bandwidth of the proposed design is due to the simple and flat structure of the radiating microstrip line. Compared to other designs the radiating element is formed by etching the microstrip line directly onto the PCB substrate above the ground plane. In this case the size of the ground plane is determined by the frequency as follows [23]:

$$
W_{S}=L_{S}=\frac{c}{2 f_{1}} \sqrt{\frac{2}{\varepsilon_{r}+1}},
$$

where $W_{S}$ and $L_{S}$ are the width and length of the ground plane, respectively; $f_{1}$ is the lowest frequency of the operation, $\varepsilon_{r}$ is the dielectric constant, and $c$ is the velocity of the light. Given the dielectric of the substrate is 10.2, therefore, using (8), the width of the antenna is approximately $16 \mathrm{~mm}$ at $4 \mathrm{GHz}$. The length of the substrate is the same as the width in order to achieve the symmetry of the structure. Figure 8 presents the simulated results of the proposed antenna with three different designs corresponding to three different sizes of ground plane. They are (i) $15 \mathrm{~mm} \times 15 \mathrm{~mm}$, (ii) $16 \mathrm{~mm} \times 16 \mathrm{~mm}$, and (iii) $17 \mathrm{~mm} \times 17 \mathrm{~mm}$. It can be seen from this figure that the lower resonant frequencies are significantly affected particularly in the first design which is also the smallest antenna. This is due to the first and second resonance frequencies being associated with the size of the p-shaped slot. The surface current tends to concentrate strongly around the p-shaped slot, thus reducing the flow of currents to a ground plane and, hence, lowering the antenna performance significantly. In general, the return loss for a second design at $-10 \mathrm{~dB}$ ranges from 4.5 to $10.9 \mathrm{GHz}$. In this frequency range the impedance bandwidth is approximately $83 \%$ compared to $80 \%$ and $78 \%$ for the first and third designs, respectively. Clearly, the second design provides a much better performance in terms of signal bandwidth while maintaining the antenna size to be as small and as compact as possible. Consequently, the second design was selected for this application.

Figure 9 compares the measured and simulated radiation patterns which are plotted in $y z$-plane. Generally, the proposed antenna exhibits a donut shape for $y z$-plane for all resonant frequencies. It can be seen from this figure that there is a good agreement between measured and computed radiation patterns within some experimental errors. In theory, the slot antenna is known as the complimentary dipole, in which the magnetic and electric fields are interchanged. Hence, it can be observed from Figure 9 that the proposed antenna has the radiation patterns which are typically the same as any typical dipole antenna at low frequencies. The pattern in the orthogonal plane ( $x y$-plane) is omnidirectional; therefore, this antenna radiates its energy equally in all directions in the $x y$-plane. Close examination of Figure 9 revealed that the radiation patterns exhibit the typical nulls, particularly at lower frequencies and at angular positions of $90^{\circ}$ and $270^{\circ}$ from the $y$-axis. The nulls in the horizontal plane can be used to protect other transmitters from interference, as it is useful in suppressing interfering signals in a given direction. From the simulated $y z$-plane pattern, the antenna has a beamwidth of $90^{\circ}$ and $64^{\circ}$, at low frequency of $4.9 \mathrm{GHz}$ and high frequency of $10.6 \mathrm{GHz}$, respectively. The beamwidth is significantly narrower for higher frequency than for lower frequency, resulting in higher gain. Hence, the amount of power radiated at high frequency is more focused and directive compared to low frequency radiations.

The simulated reflection coefficient and $\left(S_{11}\right)$ of the proposed wide-slot antenna are plotted in Figure 10, along with the equivalent measured results. This figure provides the evidence of wideband characteristic of the design, defined at threshold limit of $-10 \mathrm{~dB}$ or less. It can be observed that a good agreement was obtained between simulated and measured data, averaging at $83 \%$ and $71 \%$ for frequency ranging from 4.5 to $10.9 \mathrm{GHz}$ and from 5.1 to $10.7 \mathrm{GHz}$, respectively. 


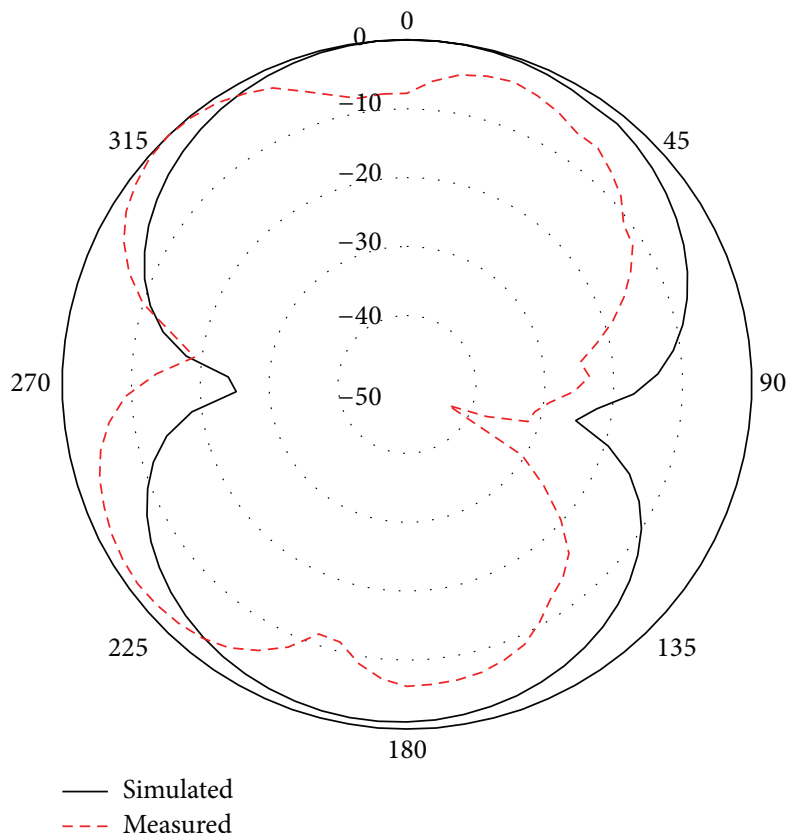

(a)

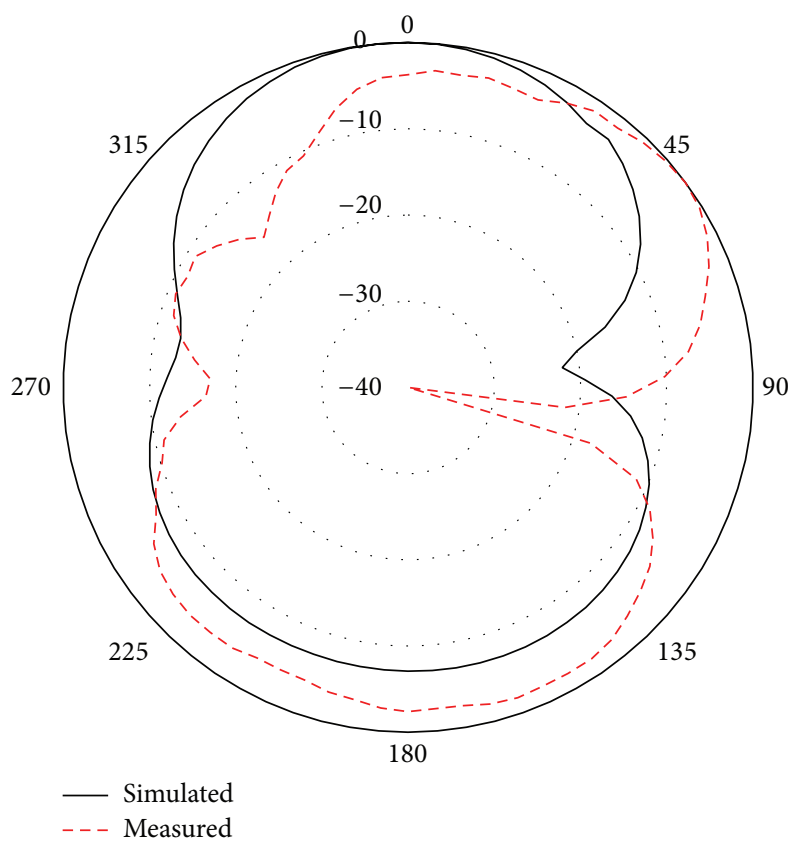

(c)

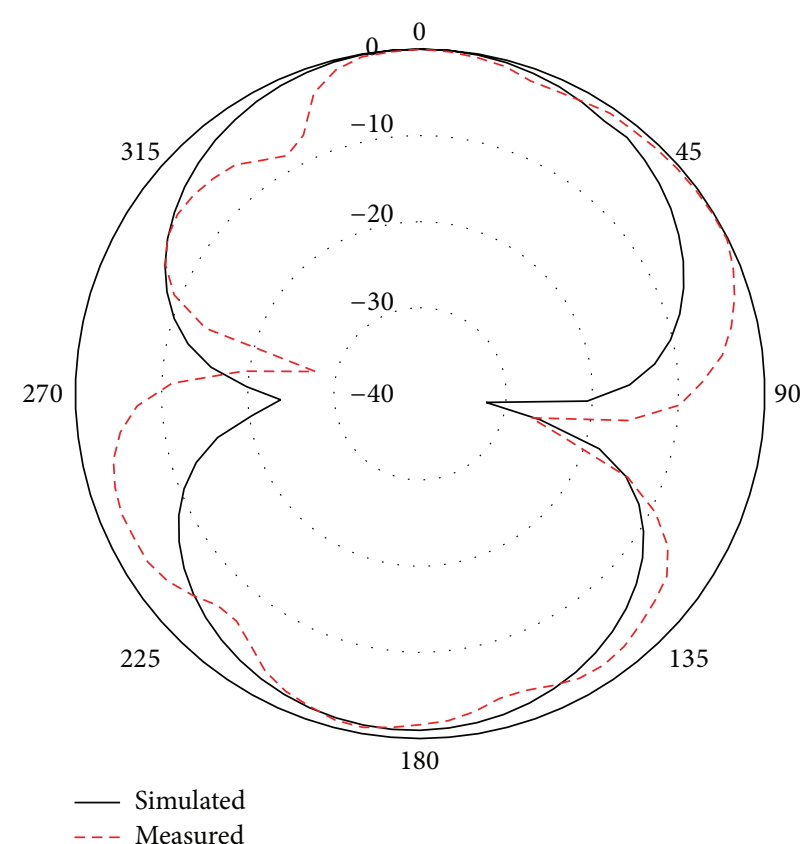

(b)

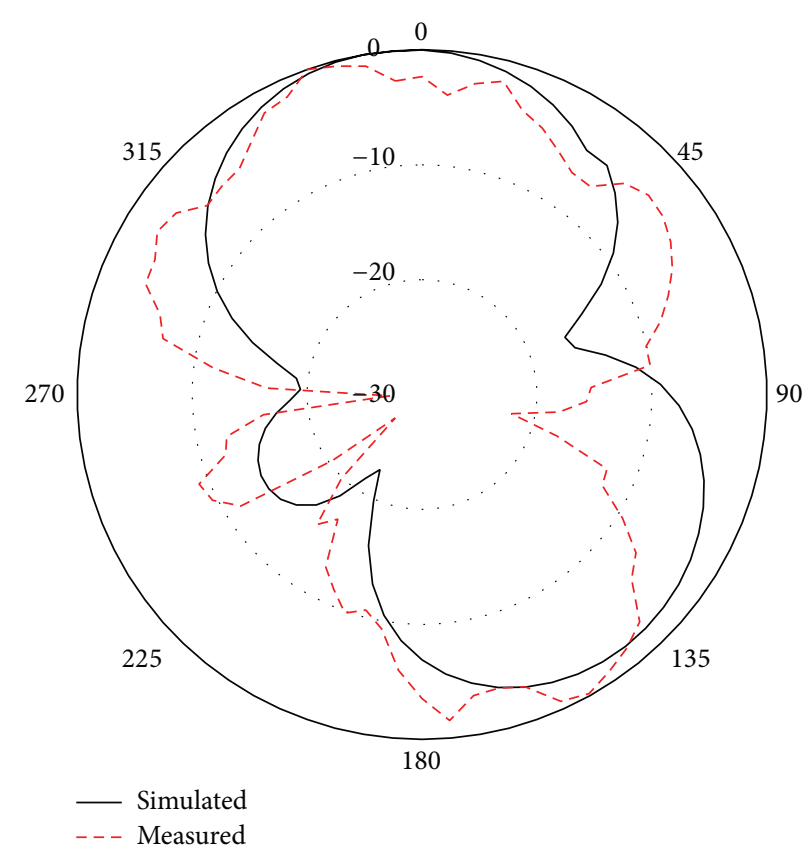

(d)

Figure 9: Simulated and measured radiation patterns at $y z$-plane at (a) $4.9 \mathrm{GHz}$, (b) $5.6 \mathrm{GHz}$, (c) $8.3 \mathrm{GHz}$, and (d) $10.6 \mathrm{GHz}$.

Both simulated and measured results demonstrate that there are four major nulls in the response. Also, the difference between the two plots is likely due to a slight mismatch in the feeding cable which is not properly modeled. There are some small discrepancies where $S_{11}$ rises above $-10 \mathrm{~dB}$ around $10 \mathrm{GHz}$ in the measured data due to the fabrication tolerances of the feed line.

The transmission coefficient $\left(S_{21}\right)$ which represents the coupling between the two sensors has also been investigated.
It is desirable to have a flat magnitude of the transmission coefficient across the desired bandwidth, in order to achieve minimal distortion for UWB signals. Figure 11 compares the simulated and measured $S_{21}$ of the proposed design. Again it can be seen that a good agreement is obtained between simulated and measured $S_{21}$ over the frequency of interest. Furthermore, it can be observed that the simulated $S_{21}$ is approximately $-20 \mathrm{~dB}$ in the frequency range of 4$11 \mathrm{GHz}$, while the measured $S_{21}$ is in the range of $-10 \mathrm{~dB}$ to 


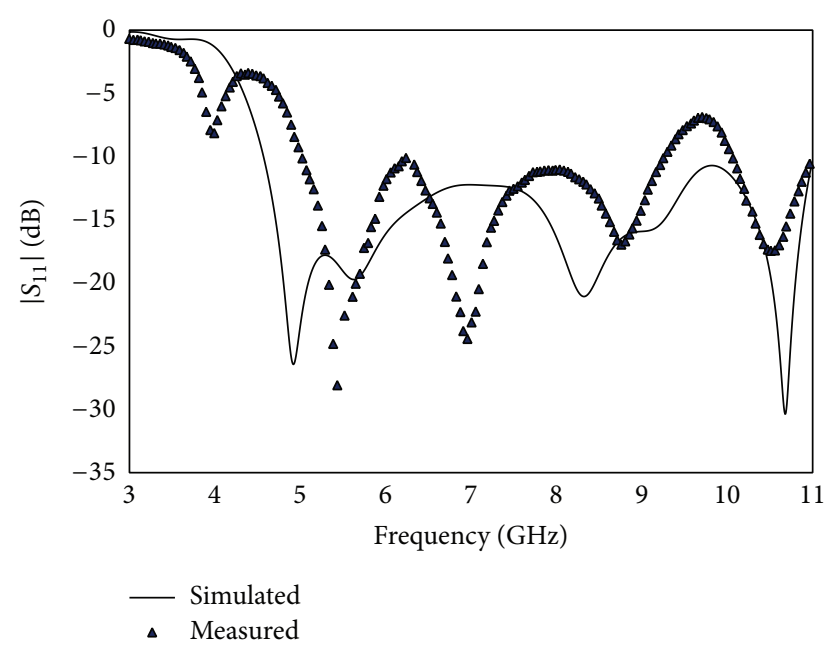

FIGURE 10: Reflection coefficients of the proposed p-shaped wideslot antenna, comparing simulated and measured values.

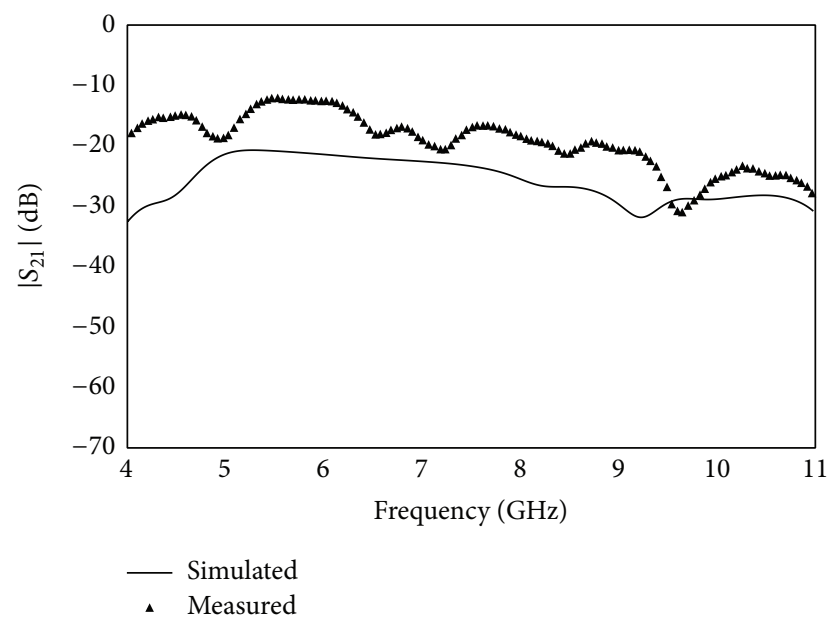

Figure 11: Transmission coefficients comparing simulated and measured values.

$-20 \mathrm{~dB}$, with variation less than $10 \mathrm{~dB}$ in this operating bandwidth. Though the proposed antenna approximately exhibits flat magnitude, several slight fluctuations were observed at $4.9 \mathrm{GHz}, 6.5 \mathrm{GHz}, 7.3 \mathrm{GHz}, 8.5 \mathrm{GHz}$, and $9.7 \mathrm{GHz}$ in the measured data. These small dips were primarily due to the effect of the SMA connector in addition to errors in measurements. Nevertheless, these discrepancies do not distort the antenna's UWB performance significantly. Additionally, the interference from multipath signals increases in near-field zone, especially in conditions where the transmitting and receiving hardware are integrated. Close examination of this figure shows that the measured and simulated $S_{21}$ is optimum at frequency of around $5 \mathrm{GHz}$ and $5.5 \mathrm{GHz}$, respectively. However, beyond $8 \mathrm{GHz}$, the measured $S_{21}$ shows more variation which indicates the reflections of the signal at the terminating end of the antenna, thus causing more dispersion at higher frequencies.

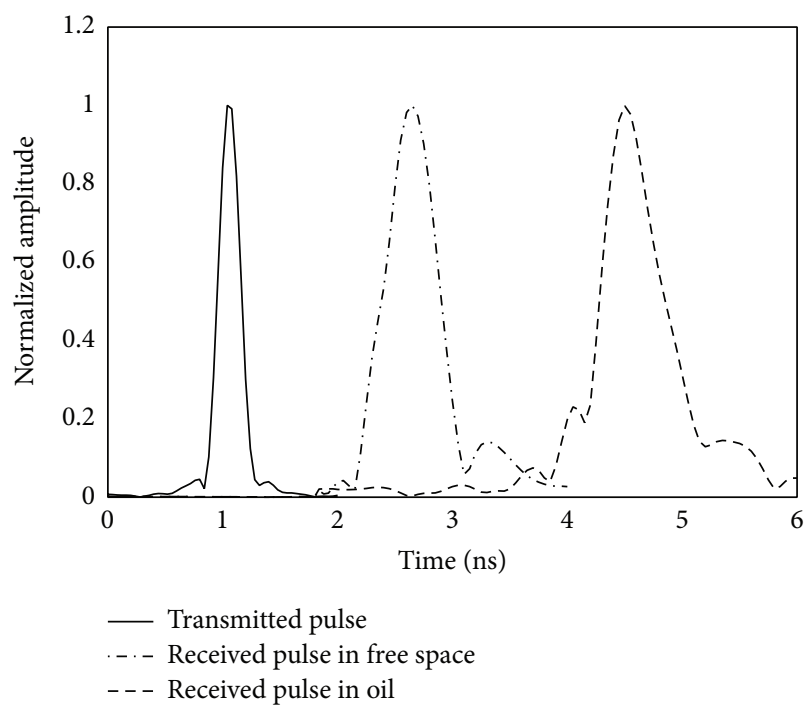

FIGURE 12: Measured response of the proposed antenna.

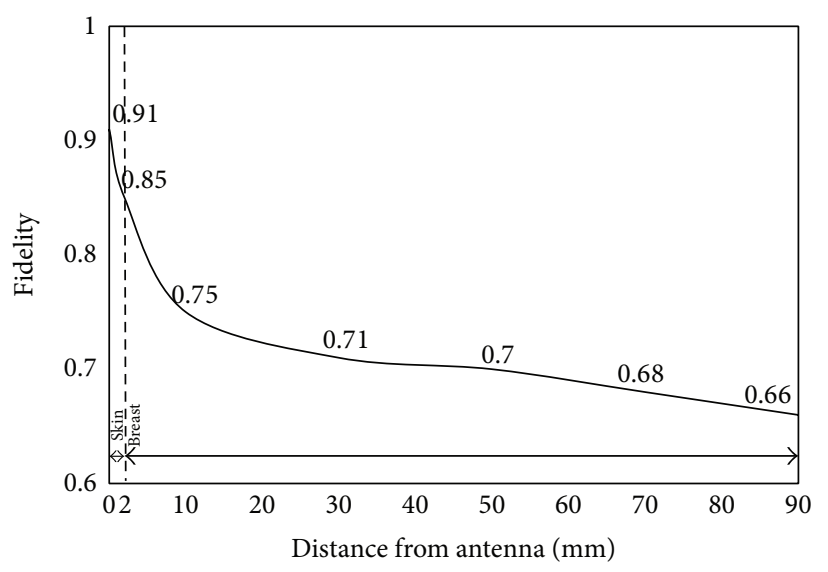

FIGURE 13: Fidelity factor as a function of distance between two antennas.

Another important characteristic of the antenna is the shape and size of pulse since this information tells general dielectric properties of the medium, particularly the scatterers. Generally, microwave detection of breast cancer relies on changes in the dielectric properties of malignant tissues as compared to healthy ones. Theoretically, the pulse shape of the signals is affected as it travels from the transmitter to target and back to receiver. For this reason the shape of the incident pulses is geometrically different from the shape of the arriving pulses. Therefore, it is important for the antenna to transmit or receive a signal with minimal distortion so that the image of the scatterer can be accurately reconstructed. In most of UWB designs, the antennas emit high and low frequency components from different portions of the geometry, so the radiated waveforms are highly distorted. This undesirable characteristic is not suitable for impulse applications including the breast cancer detection. It is pertinently important for the design to fulfill the requirement of having phase center that does not change with frequency so that the 


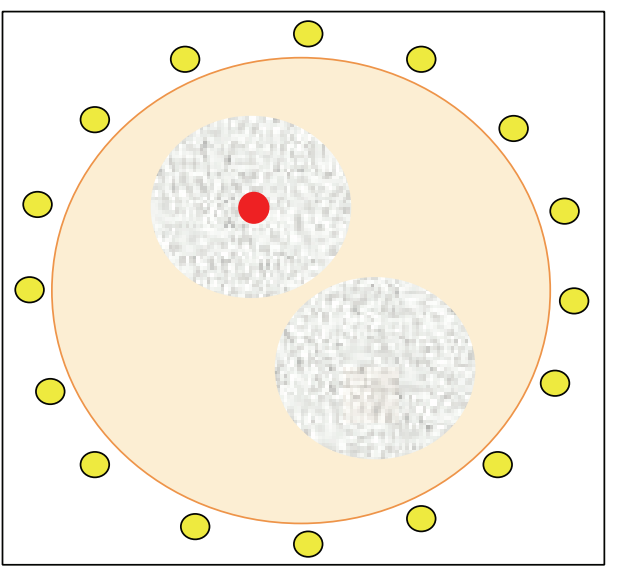

(a)

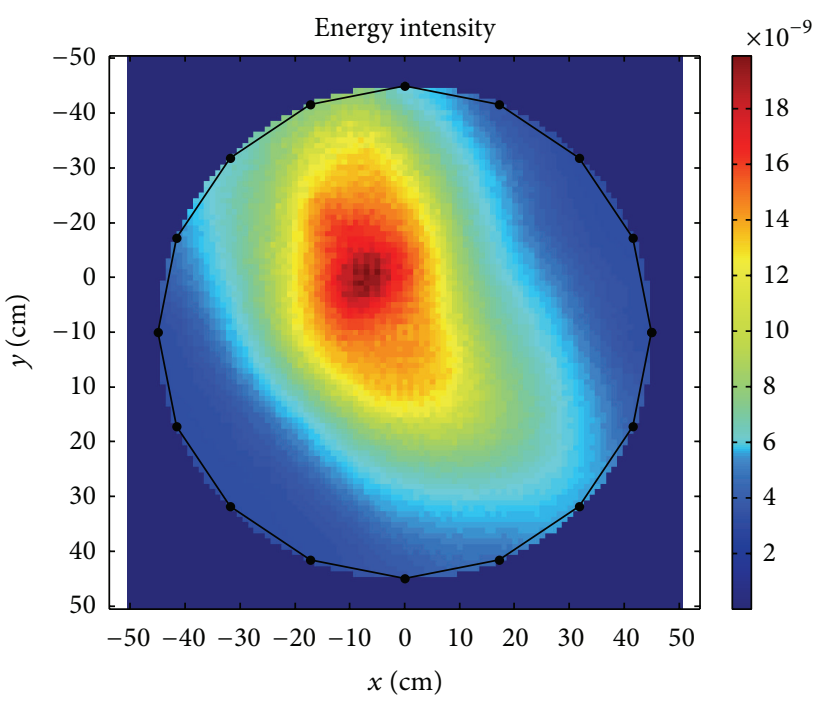

(b)

FIGURE 14: Image reconstruction of the heterogeneously dense breast phantom. (a) Actual image. (b) Image reconstructed using EDAS.

radiated emissions are minimally distorted. In order to assess the performance of the proposed antenna in transmission and reception of narrow pulses in distortionless condition, the time domain impulse response of the antenna was measured. The measurement was performed in nearly lossless media using a vegetable oil as the homogeneous background. In this way the background interferences especially the air-skin interface are minimized. Furthermore, the oil-based medium provides the best performance in terms of matching media and coupling material [24]. In the experiments, two antennas were located in the same height and separated by a fixed distance of $90 \mathrm{~mm}$. The same experiment was repeated in a free space serving as reference for oil-based measurement. In both cases the pulse duration was set to $0.6 \mathrm{~ns}$ and the data were captured for $6 \mathrm{~ns}$. The data were normalized and plotted in Figure 12. It can be observed that the pulse distortion occurred at both the falling and rising edges. In the case of transmitted pulse, pulse distortions occur at $0.8 \mathrm{~ns}$ and $1.3 \mathrm{~ns}$, with the distortion averaging at $4.1 \%$. For the received case, the distortions occur at $2.1 \mathrm{~ns}$ and $3.3 \mathrm{~ns}$, corresponding to an average distortion of $9.0 \%$. Clearly the distortions are insignificantly low in both cases and can be neglected. For the vegetable oil experiment, the pulse was delayed by $1.7 \mathrm{~ns}$ due to reduced speed of the propagation. In this case the pulse distortion occurs at $4.1 \mathrm{~ns}$ and 5.4 ns. Overall, in these experiments the distortion level is consistently less than $18.7 \%$. Even though the distortion is high in oil than free space, the overall shape of size of pulse is still preserved. This result shows that the proposed antenna retained the timing characteristics of the waveform before and after transmission. This is especially the case for sensing application with strong signals since the level of distortion is not sufficiently high to cause the pulse packets at the receiver to be changed into a long envelope that corrupts the fall and rise times excessively. Hence, the proposed design offers one significant advantage in which the timing characteristic of a pulse is nearly preserved before and after transmission.

Commonly in radar-based imaging, the ideal pulse produced by the antenna must be as short as possible with minimal late-time ringing [20]. This is to ensure that a sufficient amount of energy is transmitted, reflected, and detected by a receiver. The pulse durations of the proposed antenna are measured for different transmitterreceiver positions in the circular array. It was discovered that the pulses produced by this antenna are relatively short, averaging at $0.56 \mathrm{~ns}$ when the transmitter and receiver are adjacently located. For other positions the pulse duration ranges from $0.6 \mathrm{~ns}$ to $0.7 \mathrm{~ns}$. As expected the pulse duration increases as the distance between transmitter and receiver increases. On average the pulse duration recorded was $0.625 \mathrm{~ns}$ which is relatively short compared to other designs [12].

Another factor used to evaluate the antenna is the fidelity factor [25]. This fidelity factor is the maximum magnitude of the cross correlation between the received and incident pulses. Normalization is generally needed in this case in order to compare the shape between received and incident pulses. Assuming the time signal of receiver is much smaller than the time signal of transmitter, then the fidelity factor is defined as follows [25]:

$$
F=\max _{n} \int_{-\infty}^{\infty} \widehat{T}_{S}(t) \widehat{R}_{S}(t+\tau) d t
$$

where $\widehat{R}_{S}(t)$ is the normalized $E$-field to unit energy and $\widehat{T}_{S}(t)$ is the input signal normalized to unit energy. The result of the cross correlation ranges from 0 to 1 . The value of 1 indicates that the received pulse with no distortion is identical 
to the incident pulse, while the value of 0 indicates that the received pulse is totally different from the transmitted pulse with severe distortion. Generally, a correlation with a value of less than 0.5 (50\%) will deliver a high distortion [26], making the pulse almost unrecognizable. Figure 13 shows the computed fidelity factor as a function of the distance between transmitter and receiver. It can be observed that the fidelity decreases as the distance increases. The fidelity factor is the highest for the adjacent antenna, averaging at 0.91 in most cases. It can be seen that the fidelity factor drops to 0.6 when the distance is increased to $90 \mathrm{~mm}$. This distance corresponds to two opposing antennas in the circular array, which is also the longest sensing path. Though there is substantial reduction in the fidelity for two oppositely located antennas, the value is still reasonably good and acceptably low.

4.2. Image Reconstruction. In order to demonstrate the feasibility of this proposed antenna in detecting breast cancer at the early stage of the disease, an experiment was performed using the heterogeneously dense breast phantoms as shown in Figure 14. Referring to this figure, the actual image consists of fatty tissues, fibroglandular cells, and a tumor buried in the glandular structure located at the north left as shown in Figure 14(a), whereas Figure 14(b) is the reconstructed image. It can be observed that both the tumor and the glandular structures can be significantly detected. However, high clutter was observed causing the difficulty in recovering the exact shape as well as the size of the scatterers. The glandular structures appear fused due to excessive image blurring and other artifacts. This is partly attributed due to the decrease in the resolution as the heterogeneity of the scatterers increases. Another problem is due to the low dielectric contrast of the heterogeneous tissues, decreasing from $3: 6$ for glandular-tofatty tissues ratio to $1.7: 1$ for tumor-to-glandular tissues ratio. A similar finding is reported when radar-based technique was used to image inhomogeneous phantoms with complex dielectric distributions [22]. Clearly, a more efficient image reconstruction algorithm and advanced signal processing technique are needed to deal with this very challenging problem.

\section{Conclusions}

This paper presents a novel compact, wideband p-shaped wide-slot antenna for mapping cancerous cells at their early stage of development inside the heterogeneously dense breast tissues. A p-shaped wide-slot antenna is implemented to obtain a good reflection coefficient that can cover a wider impedance bandwidth, which is an important requirement for this type of application. The proposed antenna exhibits a wide impedance bandwidth averaging at $71 \%$ in the frequency range from 4.5 to $10.9 \mathrm{GHz}$. The antenna is printed on a square shaped Rogers' substrate RT6010 of size $16 \mathrm{~mm} \times 16 \mathrm{~mm}$ and thickness $1.27 \mathrm{~mm}$. This design also exhibits relatively distortionless characteristic with pulse width averaging at 0.625 ns. Meanwhile the value of fidelity factor is within the acceptable limits $(>0.5)$ although it decreases slightly as the distance between transmitter and receiver increases.
More importantly the proposed antenna is structurally small, compact, and planar, making it an ideal tool for breast sensing application. Image reconstruction performed using EDAS revealed that the sensors and instrumentation are capable of detecting and resolving cancerous cells located inside the fibroglandular tissue of the heterogeneous breast phantom in spite of the presence of some artifacts in the image data. These anomalies are due to cluttering and blurring which contaminated the measurement and introduced errors in the reconstruction. Further development is needed in order to improve the overall sensitivity of the system by reducing image cluttering and increasing the image-to-noise ratio.

\section{Conflict of Interests}

The authors declare that there is no conflict of interests regarding the publication of this paper.

\section{Acknowledgment}

This project was supported by USM Fundamental Research Grant Scheme 6071222.

\section{References}

[1] Federal Communications Commission, "Revision of part 15 of the commission' s rules regarding UWB transmission systems first order and report, FCC 02-48," Tech. Rep., FCC, Washington, DC, USA, 2002.

[2] C. Gilmore, P. Mojabi, A. Zakaria, S. Pistorius, and J. Lovetri, "On super-resolution with an experimental microwave tomography system," IEEE Antennas and Wireless Propagation Letters, vol. 9, pp. 393-396, 2010.

[3] E. Fear and M. Stuchly, "Microwave breast tumor detection: antenna design and characterization," in Proceeding of the IEEE Antennas and Propagation Society International Symposium, pp. 1076-1079, IEEE, Salt Lake City, Utah, USA, July 2000.

[4] S. C. Hagness, A. Taflove, and J. E. Bridges, "Wideband ultra low reverberation antenna for biological sensing," Electronics Letters, vol. 33, no. 19, pp. 1594-1595, 1997.

[5] M. Fernández Pantoja, S. González García, M. HernándezLópez, A. Rubio Bretones, and R. Gómez Martín, "Design of an ultra-broadband $\mathrm{V}$ antenna for microwave detection of breast tumors," Microwave and Optical Technology Letters, vol. 34, no. 3, pp. 164-166, 2002.

[6] X. Li, S. C. Hagness, M. K. Choi, and D. W. van der Weide, "Numerical and experimental investigation of an ultrawideband ridged pyramidal horn antenna with curved launching plane for pulse radiation," IEEE Antennas and Wireless Propagation Letters, vol. 2, pp. 259-262, 2003.

[7] W. C. Khor, M. E. Bialkowski, A. Abbosh, N. Seman, and S. Crozier, "An ultra wideband microwave imaging system for breast cancer detection," IEICE Transactions on Communications, vol. 90, no. 9, pp. 2376-2381, 2007.

[8] R. Nilavalan, I. J. Craddock, A. Preece, J. Leendertz, and R. Benjamin, "Wideband microstrip patch antenna design for breast cancer tumour detection," IET Microwaves, Antennas and Propagation, vol. 1, no. 2, pp. 277-281, 2007. 
[9] H. G. Schantz, "UWB magnetic antennas," in Proceeding of the IEEE Antennas and Propagation Society International Symposium, vol. 3, pp. 604-607, IEEE, Columbus, Ohio, USA, June 2003.

[10] M. Bod, H. R. Hassani, and M. M. S. Taheri, "Compact UWB printed slot antenna with extra bluetooth, GSM, and GPS bands," IEEE Antennas and Wireless Propagation Letters, vol. 11, pp. 531-534, 2012.

[11] S. R. Emadian, C. Ghobadi, J. Nourinia, M. H. Mirmozafari, and J. Pourahmadazar, "Bandwidth enhancement of CPW-Fed circle-like slot antenna with dual band-notched characteristic," IEEE Antennas and Wireless Propagation Letters, vol. 11, pp. 543546, 2012.

[12] D. Gibbins, M. Klemm, I. J. Craddock, J. A. Leendertz, A. Preece, and R. Benjamin, "A comparison of a wide-slot and a stacked patch antenna for the purpose of breast cancer detection," IEEE Transactions on Antennas and Propagation, vol. 58, no. 3, pp. 665-674, 2010.

[13] R. Chair, A. A. Kishk, K. F. Lee, C. E. Smith, and D. Kajfez, "Microstrip line and CPW fed ultra wideband slot antennas with U-shaped tuning stub and reflector," Progress in Electromagnetics Research, vol. 56, pp. 163-182, 2006.

[14] S.-L. S. Yang, A. A. Kishk, and K.-F. Lee, "Wideband circularly polarized antenna with L-shaped slot," IEEE Transactions on Antennas and Propagation, vol. 56, no. 6, pp. 1780-1783, 2008.

[15] J.-Y. Jan and J.-W. Su, "Bandwidth enhancement of a printed wide-slot antenna with a rotated slot," IEEE Transactions on Antennas and Propagation, vol. 53, no. 6, pp. 2111-2114, 2005.

[16] X. Qing, M. Y. W. Chia, and X. Wu, "Wide-slot antenna for UWB applications," in Proceeding of the IEEE Antennas and Wireless Propagation Society International Symposium, vol. 1, pp. 834-837, IEEE, Columbus, Ohio, USA, June 2003.

[17] S. S. Tiang, M. Sadoon, T. F. Zanoon, M. F. Ain, and M. Z. Abdullah, "Radar sensing featuring biconical antenna and enhanced delay and sum algorithm for early stage breast cancer detection," Progress in Electromagnetics Research B, no. 46, pp. 299-316, 2013.

[18] E. Porter, J. Fakhoury, R. Oprisor, M. Coates, and M. Popovic, "Improved tissue phantoms for experimental validation of microwave breast cancer detection," in Proceeding of the 4th European Conference on Antennas and Propagation (EuCAP'10), pp. 1-5, IEEE, Barcelona, Spain, April 2010.

[19] M. Lazebnik, E. L. Madsen, G. R. Frank, and S. C. Hagness, "Tissue-mimicking phantom materials for narrowband and ultrawideband microwave applications," Physics in Medicine and Biology, vol. 50, no. 18, pp. 4245-4258, 2005.

[20] S. C. Hagness, A. Taflove, and J. E. Bridges, "Two-dimensional FDTD analysis of a pulsed microwave confocal system for breast cancer detection: fixed-focus and antenna-array sensors," IEEE Transactions on Biomedical Engineering, vol. 45, no. 12, pp. 14701479, 1998.

[21] K. W. Hollman, K. W. Rigby, and M. O'Donnell, "Coherence factor of speckle from a multi-row probe," in Proceedings of the IEEE Ultrasonic Symposium, pp. 1257-1260, Caesars Tahoe, Nev, USA, October 1999.

[22] M. Klemm, J. A. Leendertz, D. Gibbins, I. J. Craddock, A. Preece, and R. Benjamin, "Microwave radar-based breast cancer detection: imaging in inhomogeneous breast phantoms," IEEE Antennas and Wireless Propagation Letters, vol. 8, pp. 1349-1352, 2009.

[23] C. A. Balanis, Antenna Theory: Analysis and Design, John Wiley \& Sons, New York, NY, USA, 1982.
[24] J. M. Sill and E. C. Fear, "Tissue sensing adaptive radar for breast cancer detection: study of immersion liquids," Electronics Letters, vol. 41, no. 3, pp. 113-115, 2005.

[25] D. Lamensdorf and L. Susman, "Baseband-pulse-antenna techniques," IEEE Antennas and Propagation Magazine, vol. 36, no. 1, pp. 20-30, 1994.

[26] O. E. Allen, D. A. Hill, and A. R. Ondrejka, "Time-domain antenna characterizations," IEEE Transactions on Electromagnetic Compatibility, vol. 35, no. 3, pp. 339-346, 1993. 

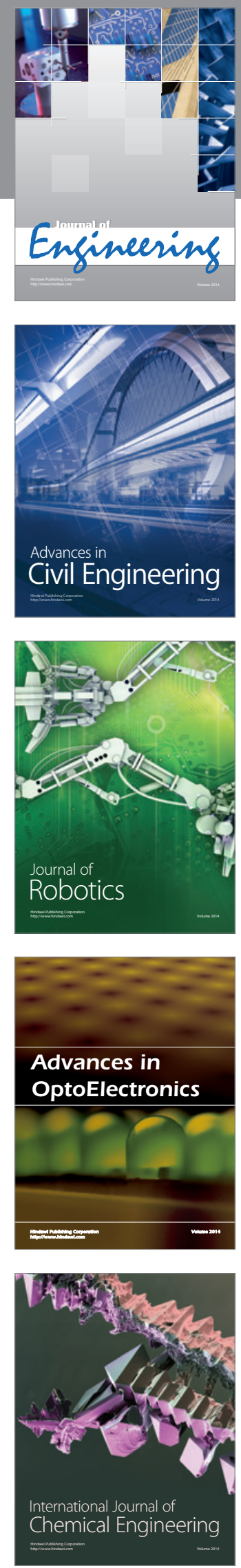

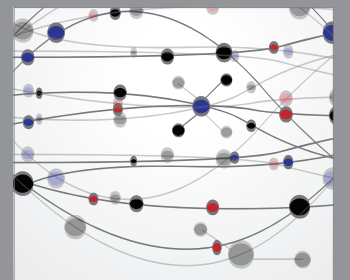

The Scientific World Journal
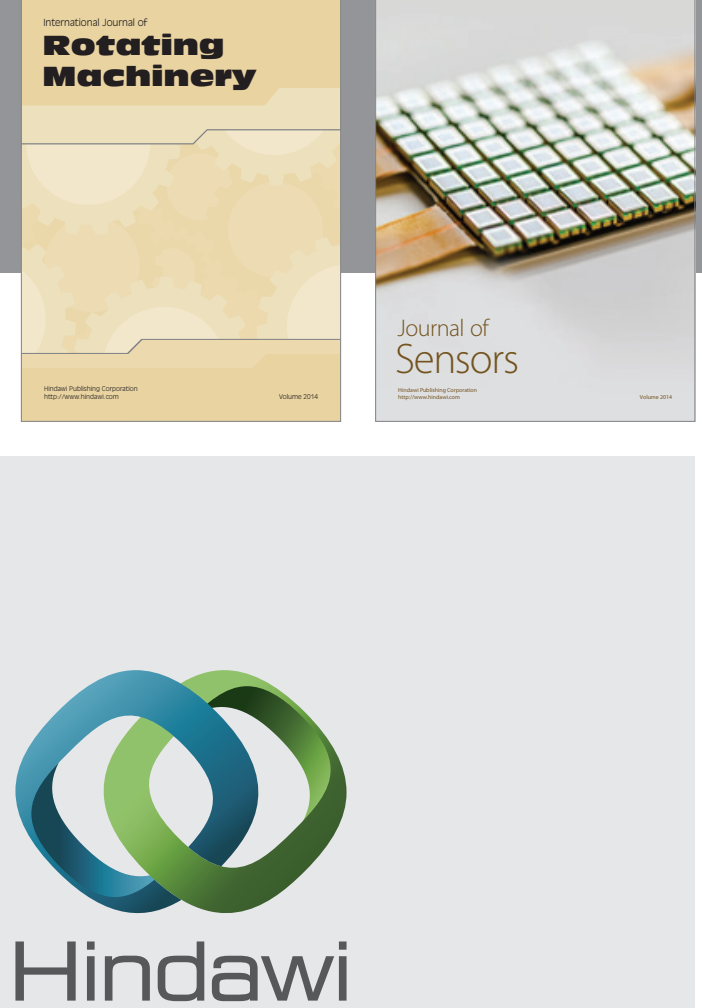

Submit your manuscripts at http://www.hindawi.com
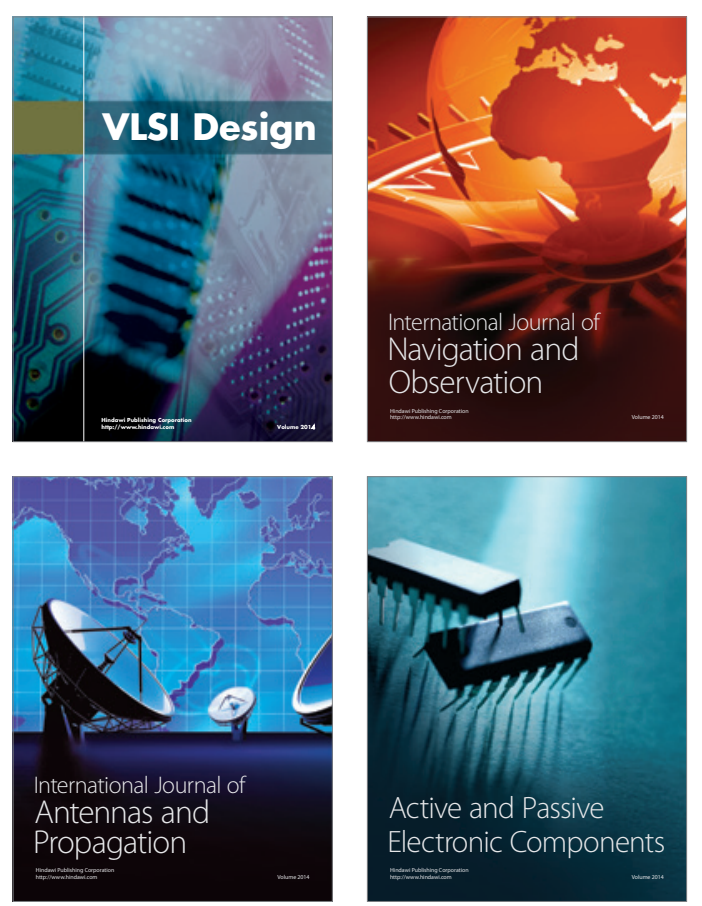
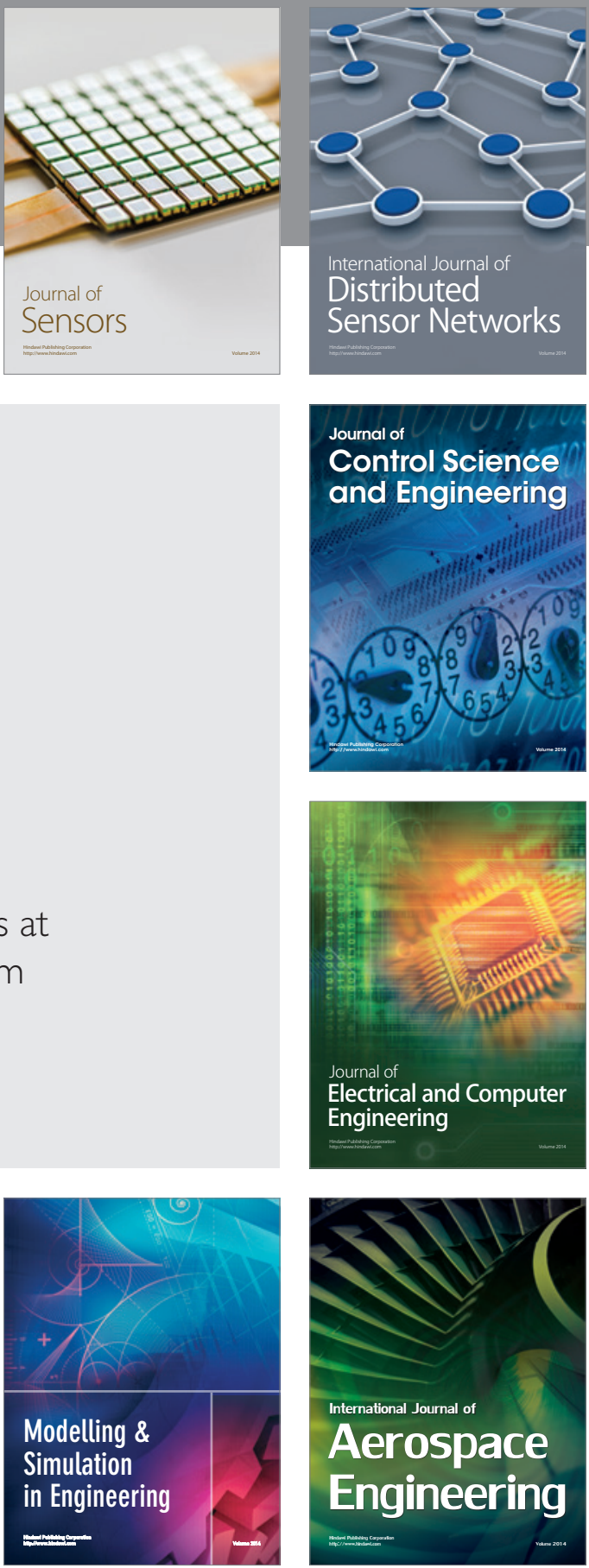

Journal of

Control Science

and Engineering
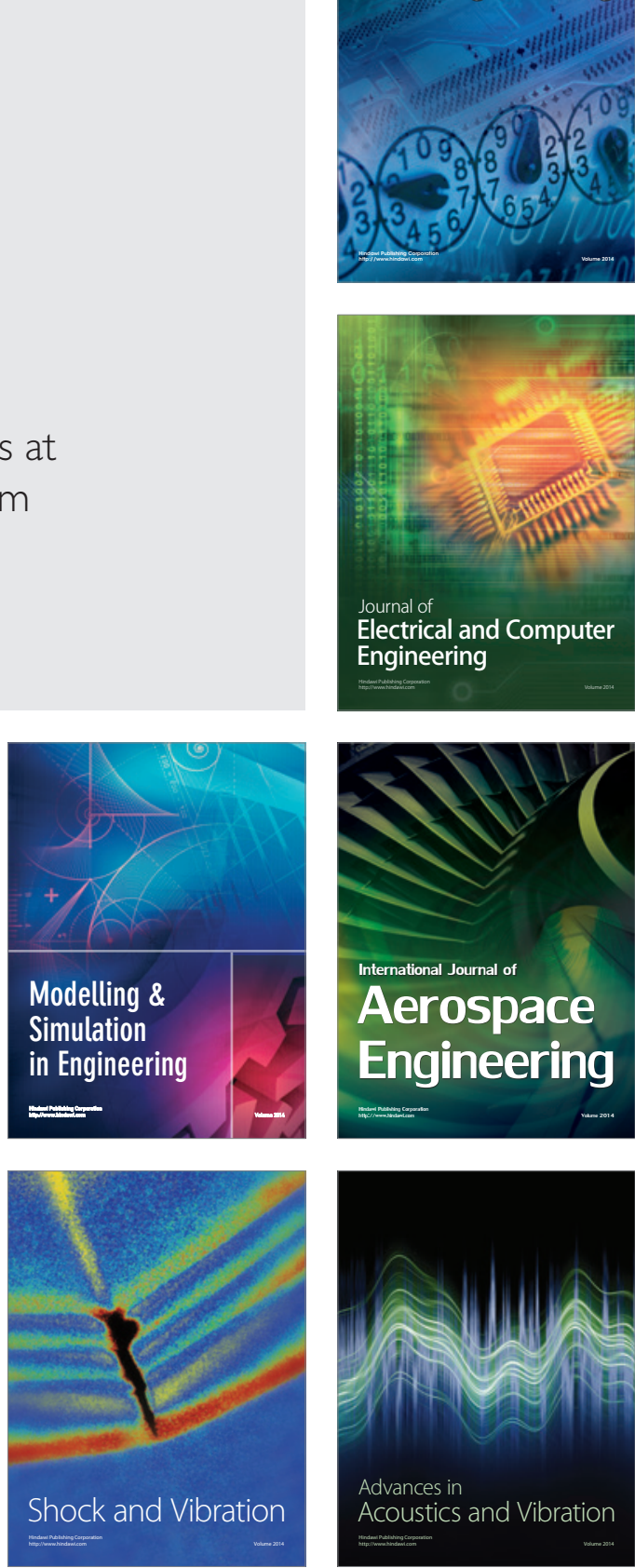